\title{
Mechanics of Biomacromolecular Networks Containing Folded Domains
}

Department of Mechanical Engineering, Massachusetts Institute of Technology, Cambridge, MA 02139 and Department of Mechanical Engineering,

University of Colorado,

Boulder, C0 80309

e-mail: qih@colorado.edu

\author{
Christine Ortiz \\ Department of Materials Science and \\ Engineering, \\ Massachusetts Institute of Technology, \\ Cambridge, MA 02139
}

\author{
Mary C. Boyce \\ Fellow ASME \\ Department of Mechanical Engineering, \\ Massachusetts Institute of Technology, \\ Cambridge, MA 02139
}

The force-extension behavior of single modular biomacromolecules is known to exhibit a characteristic repeating pattern of a nonlinear rise in force with imposed displacement to a peak, followed by a significant force drop upon reaching the peak. This "saw-tooth" pattern is a result of stretch-induced unfolding of modules along the molecular chain and is speculated to play a governing role in the function of biological materials and structures. In this paper, constitutive models for the large strain deformation of networks of modular macromolecules are developed building directly from statistical mechanics based models of the single molecule force-extension behavior. The proposed twodimensional network model has applicability to biological membrane skeletons and the three-dimensional network model emulates cytoskeletal networks, natural fibers, and soft biological tissues. Simulations of the uniaxial and multiaxial stress-strain behavior of these networks illustrate the macroscopic membrane and solid stretching conditions which activate unfolding in these microstructures. The models simultaneously track the evolution in underlying microstructural features with different macroscopic stretching conditions, including the evolution in molecular orientation and the forces acting on the constituent molecular chains and junctions. The effect of network pretension on the stress-strain behavior and the macroscopic stress and strain conditions which trigger unfolding are presented. The implications of the predicted stress-strain behaviors on a variety of biological materials are discussed. [DOI: 10.1115/1.2345442]

Keywords: protein unfolding, biological membranes, protein networks, cytoskeleton networks, mechanical properties, constitutive model

\section{Introduction}

Numerous biomacromolecules, most notably proteins, have a multi-domain architecture composed of folded modules (each typically a few $\mathrm{nm}$ in size) covalently linked to form a modular macromolecular chain [1] (Fig. 1). Each folded domain may exhibit a variety of noncovalent interactions. Unfolding of domains can be triggered by different sources including temperature, chemical denaturants, nonphysiological solvent, $p \mathrm{H}$ conditions, and mechanical loading conditions. Single molecule forceextension tests, utilizing atomic force microscopy as well as optical tweezers, have quantified the mechanically induced unfolding of folded domains. The force versus extension behavior exhibits a "saw-tooth" profile, so named due to the characteristic repeating pattern of a nonlinear rise in force to a peak, followed by a force drop after each peak (e.g., [2-4]). The nonlinear force versus extension behavior corresponds to molecular chain extension and a concomitant decrease in configurational entropy. When a critical force or energy condition is reached within a folded domain, unfolding of that domain is initiated leading to an increase in configurational entropy and a distinct drop in load. This phenomenon repeats itself as each domain along the chain unfolds. Many protein molecules have been shown to exhibit this behavior including, for example, the muscle protein titin [2]; the extracelluar matrix protein tenascin [3]; the red blood cell cytoskeleton protein spectrin [5]; and lustrin A, the protein of the nacre matrix of abalone shells [6]. Refolding of modules has been observed to occur upon removal of the applied load, the extent of which de-

\footnotetext{
${ }^{1}$ To whom correspondence should be addressed.

Contributed by the Materials Division of ASME for publication in the JournaL OF EngineEring Materials And Technology. Manuscript received November 4, 2005; final manuscript received April 27, 2006. Review conducted by Richard B. Hall. Paper presented at the 2005 ASME International Mechanical Engineering Congress (IMECE2005), November 5-11, 2005, Orlando, Florida, USA.
}

pends on the recovery time after being unloaded [1,5], but does not occur if the chain is extended by at least $42 \%$ of its contour length [7].

The mechanical response of modular macromolecules and assemblies is particularly important in systems where they are the primary structural element. For example, titin stiffness and extensibility are contributors to the passive elasticity of muscle [8]; the spectrin network is responsible for shear stiffness and the resilient elasticity of the red blood cell membrane (e.g., [9]); the protein matrix of nacre is critical to the toughening mechanisms of this biocomposite $[6,10,11]$; and the stiffness combined with large extension and remarkable toughness of spider silk are enabled by its underlying networked protein molecular architecture [12]. In the membrane and solid states, these molecules are known to be in a state of pretension [13]; the role of pretension together with potential unfolding during physiological loading conditions is not well understood. A theoretical foundation that describes the behavior of biological membranes and solids composed of modular polymers enables the development of a fundamental understanding of structure-mechanical property relationships of natural materials, soft tissues, and cells.

In this paper, single modular macromolecular chains are incorporated into physiological multi-chain assemblies to enable the simulation of larger-scale mechanical behavior of biological systems. Starting at the single macromolecule level, the freely jointed chain (FJC) and worm-like-chain (WLC) models are employed to represent the entropic elasticity of the individual polymer chains. An Eyring model is then used to capture the rate dependency of the unfolding force during single molecule extension. This single macromolecule nanomechanical behavior is integrated into one-, two-, and three-dimensional macromolecular assemblies, including: (1) a multi-macromolecular "strand," (2) an initially isotropic, surface network of molecules representing protein-rich membranes such as the spectrin network of red blood cells; and (3) an 


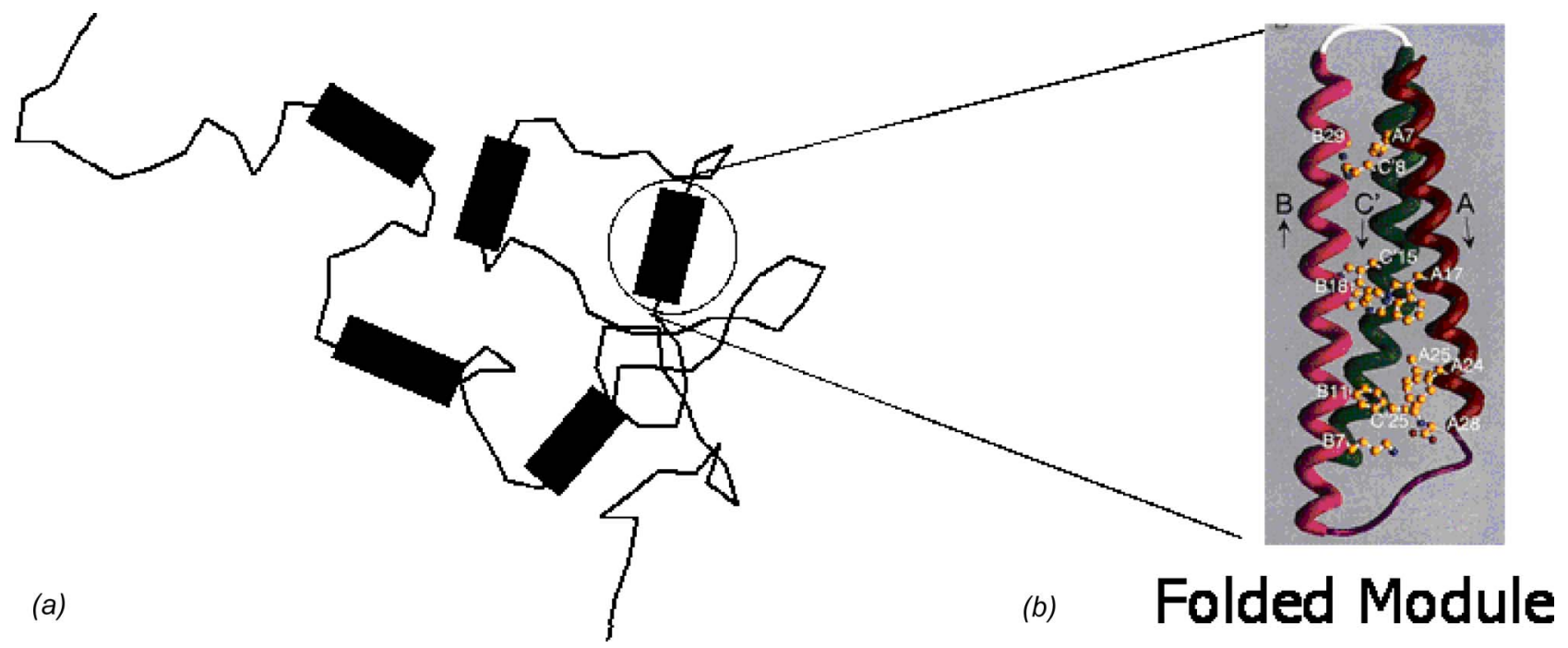

Fig. 1 (a) Schematic of a single modular polymer chain where the black rectangles represent folded modules which are connected by unfolded regions. (b) A representative folded module; here, structure of an individual Drosophilia $\alpha$-spectrin segment 14 repeat domain which exhibits a three-helix bundle [1].

initially isotropic, three-dimensional network which serves to model protein-rich networks and solids such as portions of the cytoskeletal network of cells and the entangled and/or crosslinked macromolecular structure of natural fibers and soft tissues. Constitutive models for the large stretch macroscopic stress versus strain behavior are formulated and used to predict general multiaxial loading conditions. The effects of pretension on the stressstrain behavior and the strains needed to induce unfolding are demonstrated using the model.

\section{Model Development}

\subsection{Single Molecule}

2.1.1 Structural Features. The representative macromolecule is modeled using both the FJC $[14,15]$ and WLC $[16-18]$ approximations. All formulations are presented using the FJC model; parallel formulations utilizing the WLC model are detailed in Appendix A. The conventional FJC structural representation consists of $N$ Kuhn segments or "rigid links," each of length $l$, for a total chain contour length of $L=N l$. For the modular polymer, the chain structure is further described to possess $m$ folded domains each containing $q$ Kuhn segments. When folded, a domain is taken to be one Kuhn segment; upon unfolding, a domain lengthens and adds $(q-1)$ segments to the total contour length of the chain. Thus, the chain contour length becomes a structural state variable that is updated after an unfolding event.

2.1.2 Force-Extension Behavior. The FJC model is based on a statistical survey of possible configurations, thus entropy, of a macromolecular chain during stretching. As the molecule is stretched, the number of configurations which can accommodate the extended chain end-to-end distance decrease, thus giving a decrease in configurational entropy, $\Delta S_{c}$. The FJC model determines the strain energy, $u_{c}$, during chain extension to be $-T \Delta S_{c}$, or:

$$
u_{c}=k_{B} T N\left(\frac{r}{L} \beta+\ln \frac{\beta}{\sinh \beta}\right)
$$

where $k_{B}$ is Boltzmann's constant, $T$ is absolute temperature, $r$ is the chain end-to-end distance, and $\mathcal{L}(\beta)=\operatorname{coth} \beta-1 / \beta$ defines the Langevin function and $\beta=\mathcal{L}^{-1}(r / L)$ is the inverse Langevin function. The inverse Langevin function $\beta$ characterizes the alignment of Kuhn segments towards the stretch direction and the corre- sponding reduction in entropy as $r$ approaches $L$. The axial force versus extension relationship is obtained via $f_{c}=d u_{c} / d r$, giving:

$$
f_{c}=\frac{N k_{B} T}{L} \beta
$$

2.1.3 Unfolding Criterion. Unfolding is introduced to the single macromolecule model by specifying a criterion to trigger unfolding of a module whereupon the molecule structural parameters including the number of folds, $m$, the effective number of rigid links, $N$, and the contour length (through $L=N l$ ) are updated. Here, following Rief et al. [2], an Eyring model $[19,20]$ is employed to capture the rate dependence of unfolding. The frequency of unfolding, ${ }^{2} \omega_{u}$, is thus given as:

$$
\omega_{u}=\omega_{u 0} \exp \left(-\frac{\Delta G_{u}-f_{c} x_{u}}{k_{B} T}\right)=\alpha_{u} \exp \left(\frac{f_{c} x_{u}}{k_{B} T}\right)
$$

where $\omega_{u 0}$ is an attempt frequency parameter for unfolding and is lumped into $\alpha_{u}$ through $\alpha_{u}=\omega_{u 0} \exp \left(-\Delta G_{u} / k_{B} T\right)$. The energy barrier, $\Delta G$, is determined by factors such as the type, number, placement, and orientation of noncovalent intramodule bonds, as well as environmental/solvent interactions. When the global extension force, $f_{c}$, is applied to the chain ends, $\Delta G$ is reduced by $f^{*} x_{u}$, where $x_{u}$ is the width of the activation barrier and $f^{*}$ is the local internal force state acting on the folded module. As demonstrated in molecular dynamics (MD) simulations [21,22], the unfolding process is a complex sequence of local events. Whether unfolding occurs due to normal or shear separation of bonds, or some combination thereof, is not clear and will depend upon the internal bond structure of a particular fold. Therefore, describing a relationship between the global extension force acting on the molecule $\left(f_{c}\right)$ and the features of the local force state (as represented by $\left.f^{*}\right)$ is not straightforward. For simplicity, $f^{*}$ is taken to be equal to $f_{c}$. The material parameters $\alpha_{u}$ and $x_{u}$ are fit to unfolding force data taken at different extension rates. The net frequency of unfolding of a module along a chain during a time period of $\Delta t$ is thus:

\footnotetext{
${ }^{2}$ Note that refolding events could easily be included by the addition of the frequency of "backwards" events term, i.e., the frequency of refolding. In this paper, we focus on monotonic loading and thus the probability of refolding events is negligible and is thus neglected.
} 


$$
p=m_{t} \omega_{u} \Delta t
$$

where $m_{t}$ is the current number of folded domains along the chain at time $t$. The implementation of the unfolding criterion is provided in Appendix B.

2.2 Modular Macromolecular Assemblies. Models for assemblies of modular polymers are presented below, including onedimensional, two-dimensional, and three-dimensional structures.

2.2.1 Multi-Molecule Strands. Here, a multi-molecule "strand" is defined as an assembly of non-interacting macromolecules extended in one direction simultaneously. We consider the simple case of a strand consisting of $k$ molecules with different initial contour lengths, but otherwise identical nanomechanical behavior arranged in parallel. The total force, $F$, required to stretch the strand is simply the summation of the axial force contribution from each molecule:

$$
F=k_{B} T \sum_{i=1}^{k} \frac{N_{i}}{L_{i}} \beta_{i}
$$

where the subscript $i$ denotes the $i$ th molecule and $\beta_{i}=\mathcal{L}^{-1}\left(r / L_{i}\right)$. The probability of unfolding is monitored for each constituent macromolecule during stretching and the contour length of the corresponding molecule is updated after an unfolding event.

2.2.2 Membrane Networks. Membranes composed of protein networks can be modeled as two-dimensional networks where the imposed stretch and the stress state lie in the surface of the membrane. One example is the network of spectrin proteins which serve as the membrane skeleton of red blood cells [23,24]. Here, a random network is modeled utilizing an idealized "four-chain" network (Fig. 2(a)) whereby four chains emanate from a central junction of an initially square patch with each chain extending to one corner of the square; the edges of the square are taken to lie normal to the principal directions of stretch. This network idealization is a two-dimensional equivalent to the three-dimensional eight-chain model of a random network [25]. The four-chain network model captures the essential features of a random network in an "average" sense whereby the network chains stretch and rotate to accommodate the imposed macroscopic deformation. The initial end-to-end length of a constituent chain, $r_{0}$, is obtained from electron micrograph images ${ }^{3}$. As shown in Fig. 2(b), the network is subjected to principal stretches within the plane, $\lambda_{1}$ and $\lambda_{2}$, which result in a stretched constituent chain length of $r=\lambda_{c} r_{0}$, where $\lambda_{c}=\left(I_{1(2 \mathrm{D})} / 2\right)^{1 / 2}$ is the chain stretch and $I_{1(2 \mathrm{D})}=\lambda_{1}^{2}+\lambda_{2}^{2}$. Furthermore, in cell membranes, we note that the surface area is typically preserved during stretching due to the two-dimensional constraint of the fluid-like lipid bilayer which is connected to the membrane skeleton (see, for example, [9,26-28]). The surface area constraint is mathematically expressed as $\lambda_{1} \lambda_{2}=1$. The strain energy of the RVE subjected to a membrane stretch is simply the sum of the strain energy in the four constituent chains due to their change in entropy:

$$
U=4 k_{B} T N\left[\frac{\lambda_{c} r_{0}}{L} \beta_{c}+\ln \left(\frac{\beta_{c}}{\sinh \beta_{c}}\right)-c\right]
$$

where $\beta_{c}=\mathcal{L}^{-1}(r / L)=\mathcal{L}^{-1}\left(\lambda_{c} r_{0} / L\right), c$ is the contribution from the initial entropy prior to external loading, $c=r_{0} \beta_{c 0} / L$ $+\ln \left(\beta_{c 0} / \sinh \beta_{c 0}\right)$, and $\beta_{c 0}=\mathcal{L}^{-1}\left(r_{0} / L\right)$. The strain energy per unit initial surface area is $\widetilde{U}=U /\left(4 a^{2}\right)$. Recognizing that the chain density (number of chains per unit initial surface area) is $\nu=4 /\left(4 a^{2}\right)$,

\footnotetext{
${ }^{3}$ Alternatively, following random walk statistics, $r_{o}$ can be taken to be $r_{0}=\sqrt{N} /$ when the network formation is a random process; this is the common assumption in rubber elasticity. The non-zero initial length of $r_{0}$ prescribes a pretension in the network chains; the pretension is balanced by equal and opposite intermolecular interactions as in rubbery solids; or by interactions with the lipid bilayer, other molecules and/or the cytosol.
}

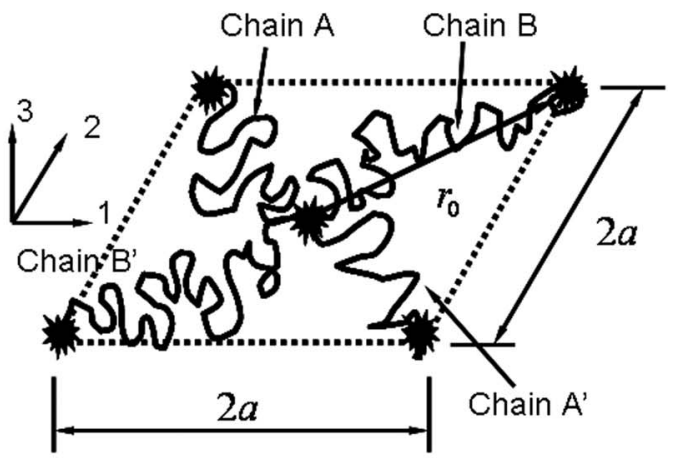

(a)

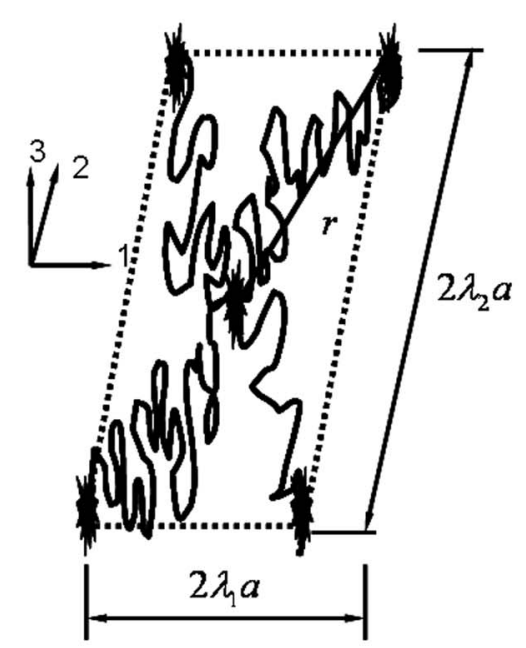

(b)

Fig. 2 Schematic of a two-dimensional network: (a) The fourchain network, $(b)$ the four-chain network after deformation

the strain energy per unit initial surface area is then:

$$
\tilde{U}=\nu k_{B} T N\left[\frac{\lambda_{c} r_{0}}{L} \beta_{c}+\ln \left(\frac{\beta_{c}}{\sinh \beta_{c}}\right)-c\right]
$$

This strain energy function is a higher-order function of $I_{1(2 \mathrm{D})}$ $=\lambda_{1}^{2}+\lambda_{2}^{2}$ as seen upon expansion of the inverse Langevin function. Including only the first-order terms (useful for small to modest deformations) recovers the two-dimensional neo-Hookean model (utilized by Skalak et al. [26] and Evans and Hochmuth [28] in their early models of the red blood cell membrane as well as recently by Dao et al. [29]). Mills et al. [30] have used a phenomenological higher-order $I_{1(2 \mathrm{D})}$ model to capture the large deformations of cell membranes. Thus, higher-order $I_{1(2 \mathrm{D})}$ models are phenomenological equivalents to molecularly based non-Gaussian statistical mechanics models (as also shown in Arruda and Boyce [25] and Boyce and Arruda [31] for three-dimensional networks). Unfolding of chains within the membrane network is captured using Eq. (7) together with the unfolding criteria; $N$ and $L$ are then updated when an unfolding event occurs, thus updating the structural state of the network and its corresponding strain energy function.

For a general membrane deformation described by the membrane deformation gradient $\mathbf{F}_{2 \mathrm{D}}=\partial \mathbf{x} / \partial \mathbf{X}$ and the membrane left Cauchy Green tensor $\mathbf{B}_{2 \mathrm{D}}=\mathbf{F}_{2 \mathrm{D}} \mathbf{F}_{2 \mathrm{D}}^{T}$, the Cauchy membrane stress is found by proper differentiation of $U$ (see Appendix $\mathrm{C}$ ) and is given by: 


$$
\widetilde{\boldsymbol{\sigma}}=\nu k_{B} T \frac{r_{0} N}{2 \lambda_{c} L} \beta_{c} \mathbf{B}_{2 \mathrm{D}}+\tilde{h} \mathbf{I}_{2 \mathrm{D}}
$$

where $\mathbf{I}_{2 \mathrm{D}}$ is the 2D identity tensor, $\lambda_{c}=\sqrt{\operatorname{tr}\left(\mathbf{B}_{2 \mathrm{D}}\right) / 2}$, and $\tilde{h}$ is the additional equibiaxial stress (due to the constant surface area constraint $\left.I_{2(2 \mathrm{D})}=\operatorname{det}\left(\mathbf{B}_{2 \mathrm{D}}\right)=\left(\lambda_{1} \lambda_{2}\right)^{2}=1\right)$ obtained by satisfying equilibrium through the traction boundary conditions. Note that here we define Cauchy membrane stress to be the product of the Cauchy stress and the membrane thickness.

The effects of distributed aspects of the network microstructure can be examined by taking the four-chain network to be constituted of two sets of chains of different initial contour length but identical initial end-to-end distance (referring to Fig. 2(a), chains $A$ and $A^{\prime}$ are taken to have initial contour length $L_{A}$; chains $B$ and $B^{\prime}$ have initial contour length $L_{B}$ ). The strain energy density expression becomes:

$$
\tilde{U}=\frac{\nu k_{B} T}{2} \sum_{i=A, B} N_{i}\left[\frac{\lambda_{c} r_{0}}{L_{i}} \beta_{c i}+\ln \left(\frac{\beta_{c i}}{\sinh \beta_{c i}}\right)\right]-c
$$

with corresponding Cauchy stress:

$$
\tilde{\boldsymbol{\sigma}}=\frac{\nu k_{B} T}{2} \sum_{i=A, B} \frac{r_{0} N_{i}}{2 \lambda_{c} L_{i}} \beta_{c i} \mathbf{B}_{2 \mathrm{D}}+\tilde{h} \mathbf{I}_{2 \mathrm{D}}
$$

where the subscript $i$ represents the parameters associated with the $i$ th molecule $(i=A, B)$ and $\beta_{c i}=\mathcal{L}^{-1}\left(r / L_{i}\right)=\mathcal{L}^{-1}\left(\lambda_{c} r_{0} / L_{i}\right)$. Note that this arrangement of chains in the four-chain network representation retains isotropic material behavior since the network is always aligned in principal stretch space. The chains with the shorter initial contour length will unfold at a smaller stretch than those with the longer initial contour length which enables a first study of the distributed nature of unfolding of macromolecules on the stress-strain behavior of a network.

2.2.3 Three-Dimensional Networks. In order to study biological solids constituted of modular macromolecules, a threedimensional network model is necessary. Here, we pursue a network modeling protocol based on rubber elasticity theory and begin with the eight-chain network model $[25,32,33]$. The eightchain network approximates a three-dimensional randomly oriented macromolecular network as a network consisting of eight chains in a cube; each chain emanates from the center of the cube to a corner of the cube (Fig. 3(a)) and the edges of the cube are aligned with the principal stretches. Upon deformation, the macromolecular chains stretch and rotate towards the maximum principal stretch axis(es) to accommodate the imposed macroscopic deformation, thus emulating the essential physics of a more complex random network. From Fig. 3(b), the chain stretch ratio $\lambda_{c}$ is $\lambda_{c}=r / r_{0}=\sqrt{I_{1} / 3}$, where $\lambda_{1}, \lambda_{2}$, and $\lambda_{3}$ are the three principal stretches, and $I_{1}=\lambda_{1}^{2}+\lambda_{2}^{2}+\lambda_{3}^{2}$. The force on each chain is obtained using FJC statistical mechanics together with the unfolding criterion. Taking the deformation to be incompressible (note this can be extended to cover the case of compressible materials by inclusion of pressure-volume contributions to the strain energy (see, for example, $[31,34])$ ), the strain energy density is given by:

$$
\bar{U}=\nu k_{B} T N\left[\frac{\lambda_{c} r_{0}}{L} \beta_{c}+\ln \frac{\beta_{c}}{\sinh \beta_{c}}-c\right]
$$

where $\nu$ is the number of macromolecular chains per unit reference volume, or chain density, $\beta_{c}=\mathcal{L}^{-1}(r / L)=\mathcal{L}^{-1}\left(\lambda_{c} r_{0} / L\right), c$ is the contribution of initial entropy of a macromolecule, and $c$ $=\sqrt{1 / 3 N} \beta_{c 0}+\ln \left(\beta_{c 0} / \sinh \beta_{c 0}\right)$, and $\beta_{c 0}=\mathcal{L}^{-1}\left(r_{0} / L\right)$. Furthermore, each chain will unfold when the unfolding criterion is satisfied which in turn updates the material parameters $N$ and $L$.

A general multiaxial deformation can be described by the deformation gradient $\mathbf{F}=\partial \mathbf{x} / \partial \mathbf{X}$, where $\mathbf{x}$ represents the current position and $\mathbf{X}$ represents the reference position, and the left Cauchy Green tensor is given by $\mathbf{B}=\mathbf{F F}^{T}$. The Cauchy stress tensor is:

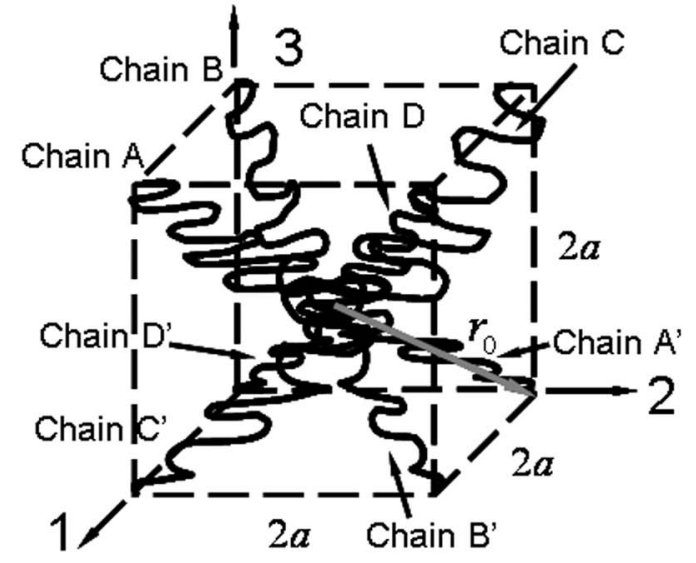

(a)

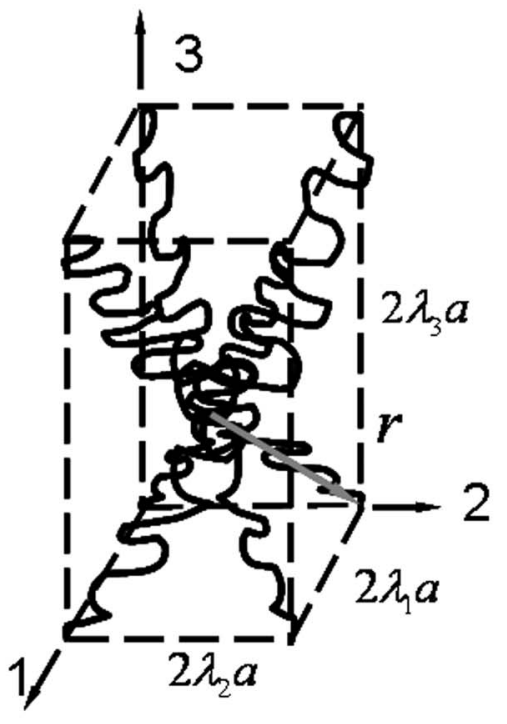

(b)

Fig. 3 Schematic of the three-dimensional eight-chain network: (a) The eight-chain network; (b) The eight-chain network after deformation

$$
\boldsymbol{\sigma}=\nu k_{B} T \frac{r_{0} N}{3 \lambda_{c} L} \beta_{c} \mathbf{B}-p^{*} \mathbf{I}
$$

where $\mathbf{I}$ is the $3 \mathrm{D}$ diagonal identity tensor, $p^{*}$ is the additional pressure (due to volume conservation constraint), and $\lambda_{c}$ $=\sqrt{\operatorname{tr}(\mathbf{B}) / 3}$.

A distribution in initial contour lengths can be examined within the context of the eight-chain network by considering those chains on the same diagonal to possess the same initial contour length but one which differs from those on other diagonals (referring to Fig. 3(a), we take the four initial contour lengths of $L_{A}$ for chains $A$ and $A^{\prime}, L_{B}$ for $B$ and $B^{\prime}, L_{C}$ for $C$ and $C^{\prime}$, and $L_{D}$ for $D$ and $D^{\prime}$ ) giving the strain energy density function:

$$
\bar{U}=\frac{\nu k_{B} T}{4} \sum_{i=A, B, C, D} N_{i}\left[\frac{\lambda_{c} r_{0}}{L_{i}} \beta_{c i}+\ln \frac{\beta_{c i}}{\sinh \beta_{c i}}\right]-c
$$

and Cauchy stress:

$$
\boldsymbol{\sigma}=\frac{\nu k_{B} T}{4} \sum_{i=A, B, C, D} \frac{r_{0} N_{i}}{3 \lambda_{c} L_{i}} \beta_{c i} \mathbf{B}-p^{*} \mathbf{I}
$$




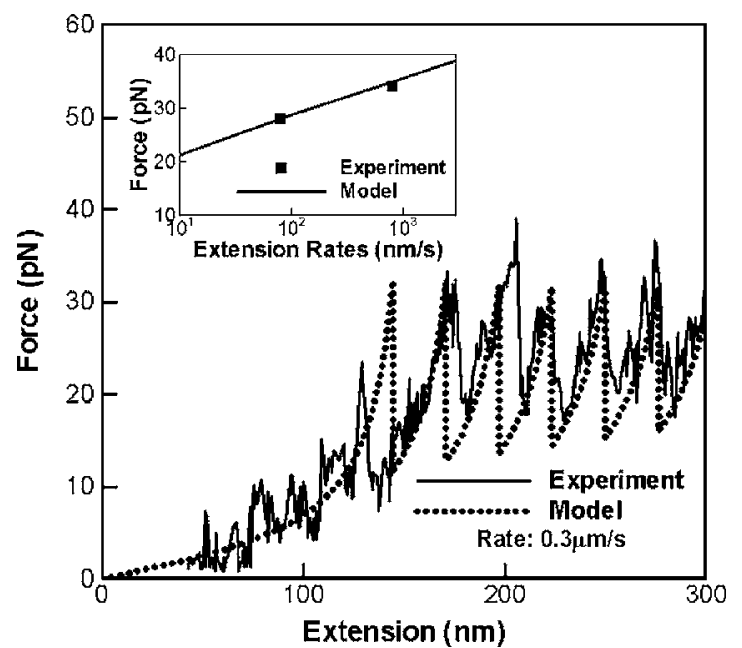

Fig. 4 FJC model compared to experiment: Spectrin force versus extension behavior; simulation uses a single valued energy barrier; experimental data from Rief et al. [2], the inset shows the dependence of unfolding force on extension rate

\section{Results}

3.1 Single Modular Macromolecule. Figure 4 compares the FJC model predictions of force versus extension to experimental data reported in the literature for single molecule native spectrin [2]. The model parameters were obtained by fitting to the experimental data of Rief et al. [5] and are given in Table 1. The model captures the overall force versus extension behavior as well as the force at which unfolding occurs for this spectrin. Similar agreement was obtained using the WLC model (see Appendix A for a direct comparison of WLC to FJC for the case of membrane deformation). The Fig. 4 inset shows the dependence of the spectrin unfolding force on extension rate, where the force needed to initiate unfolding increases with increasing extension rate.

3.2 Multi-Molecule Strands. Figure 5 shows the simulation results for the normalized force versus extension behavior for non-interacting molecular strands composed of 5 and 20 molecules compared to single molecule behavior. The total force values were normalized by the number of molecules in the strand. The initial contour lengths of constituent chains were taken to have a small distribution of $174 . \pm 26$. $\mathrm{nm}$. The force-extension behavior of strands containing multiple molecules shows numerous peaks with smaller peak values and smaller force drops after each peak than the single molecule behavior. The rather small distribution in initial contour length thus results in a plateau-like force behavior with increasing numbers of molecules in contrast to the discrete saw-tooth profile of the single molecule behavior. This indicates that small variations in assemblies of modular mol-

Table 1 Modular chain structure and properties

\begin{tabular}{|c|c|c|c|}
\hline Model parameters & $\begin{array}{r}\text { Spe } \\
\text { single } \\
\text { WLC }\end{array}$ & $\begin{array}{l}\text { in, } \\
\text { lecule } \\
\text { FJC }\end{array}$ & $\begin{array}{c}\text { Spectrin, } \\
\text { networked } \\
\text { heterodimer } \\
\text { molecule } \\
\text { FJC }\end{array}$ \\
\hline $\begin{array}{l}\text { Persistence length, } \mathrm{s},(\mathrm{WLC}) \text { or } \\
\text { statistical segment length (FJC), } l(\mathrm{~nm})\end{array}$ & 0.8 & 1.6 & 5.13 \\
\hline Initial contour length $(\mathrm{nm}), L_{0}$ & 197. & 174. & 180 \\
\hline $\begin{array}{l}\text { Increase in contour length due } \\
\text { to unfolding, } \Delta L(\mathrm{~nm})\end{array}$ & 32.0 & 28.8 & 28.8 \\
\hline Activation barrier width, $x_{u}(\mathrm{~nm})$ & 1.7 & 1.7 & 1.7 \\
\hline$\alpha\left(\times 10^{-6} \mathrm{~s}^{-1}\right)$ & 6.0 & 6.0 & 6.0 \\
\hline
\end{tabular}

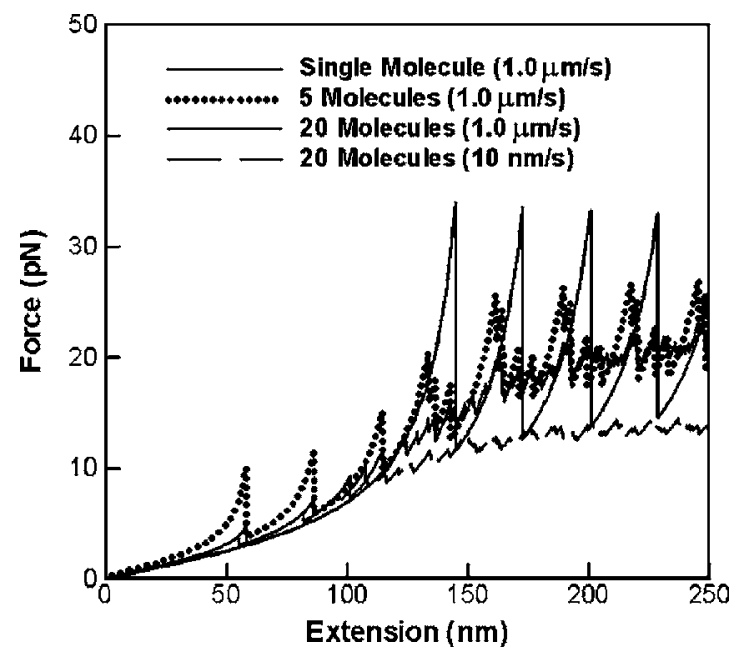

Fig. 5 Force versus extension curves from simulations of stretching multimolecule. The force is reported as force per unit molecule.

ecules can provide a plateau region in force-extension behavior The plateau may be a mechanism for controlling load transfer within a biological structure and/or enabling larger scale deformation and structural rearrangements under relatively constant force conditions.

3.3 Macromolecular Membrane Network Model. The stress-stretch behavior of a membrane is simulated under conditions of uniaxial tension and simple shear. The material properties used in the simulation are listed in Table 1. The initial chain end-to-end distance between cross-link sites was taken to be $r_{0}$ $=75 \mathrm{~nm}[23,35]$ and is representative of the distance between cross-links in the spectrin skeletal network. Due to the geometry of the network, the chain density $\nu$ is calculated to be $3.6\left(10^{14}\right) \mathrm{m}^{-2}$. The initial contour length is taken to be nominally $180 \mathrm{~nm}$ based on micrographs of spread membranes which depict fully extended chains in the spectrin network (Liu et al. [24]); $L_{0}$ is observed to be distributed over a range from approximately $150-210 \mathrm{~nm}$. The Kuhn length was then chosen to be $5.13 \mathrm{~nm}$ to best fit the observed shear modulus $\left(\sim 0.6-1.0\left(10^{5}\right) \mathrm{N} / \mathrm{m}[9]\right)$ of the spectrin network ${ }^{4}$. Unfolding is triggered using Eq. (4).

Figure 6(a) shows the simulated rate-dependent uniaxial stressstretch behavior for the case of a uniform network $\left(L_{0}\right.$ of all chains being $180 \mathrm{~nm}$ ). As shown, a decrease in strain rate is observed to result in a decrease in the strain at which first unfolding occurs, and thus a decrease in the unfolding stress and the magnitude of the stress drop upon unfolding. At the lowest strain rate shown $(0.01 / \mathrm{s})$, the unfolding stresses and corresponding drops are barely noticeable and, indeed, a stress-stretch behavior more characteristic of a traditional compliant rubber-like behavior is observed with a softening response that is somewhat hidden when observing monotonic stretching. The peak nominal stress is also seen to be relatively constant. A distribution in the initial contour length acts to smooth and flatten out the curves, decreasing the sharpness of the stress peaks and the magnitude of the stress drops. This suggests that a distributed network microstructure provides a plateau in the nominal stress-strain behavior of a network due to the multitude of unfolding events, each occurring at slightly different stretches.

${ }^{4}$ The Kuhn length for the networked spectrin is approximately the length of a folded domain, whereas that used in fits of the single molecule tests is approximately the residue length; we speculate this difference to be due to the heterodimer structure of the networked spectrin as well as due to the preparation of single molecules for testing. 

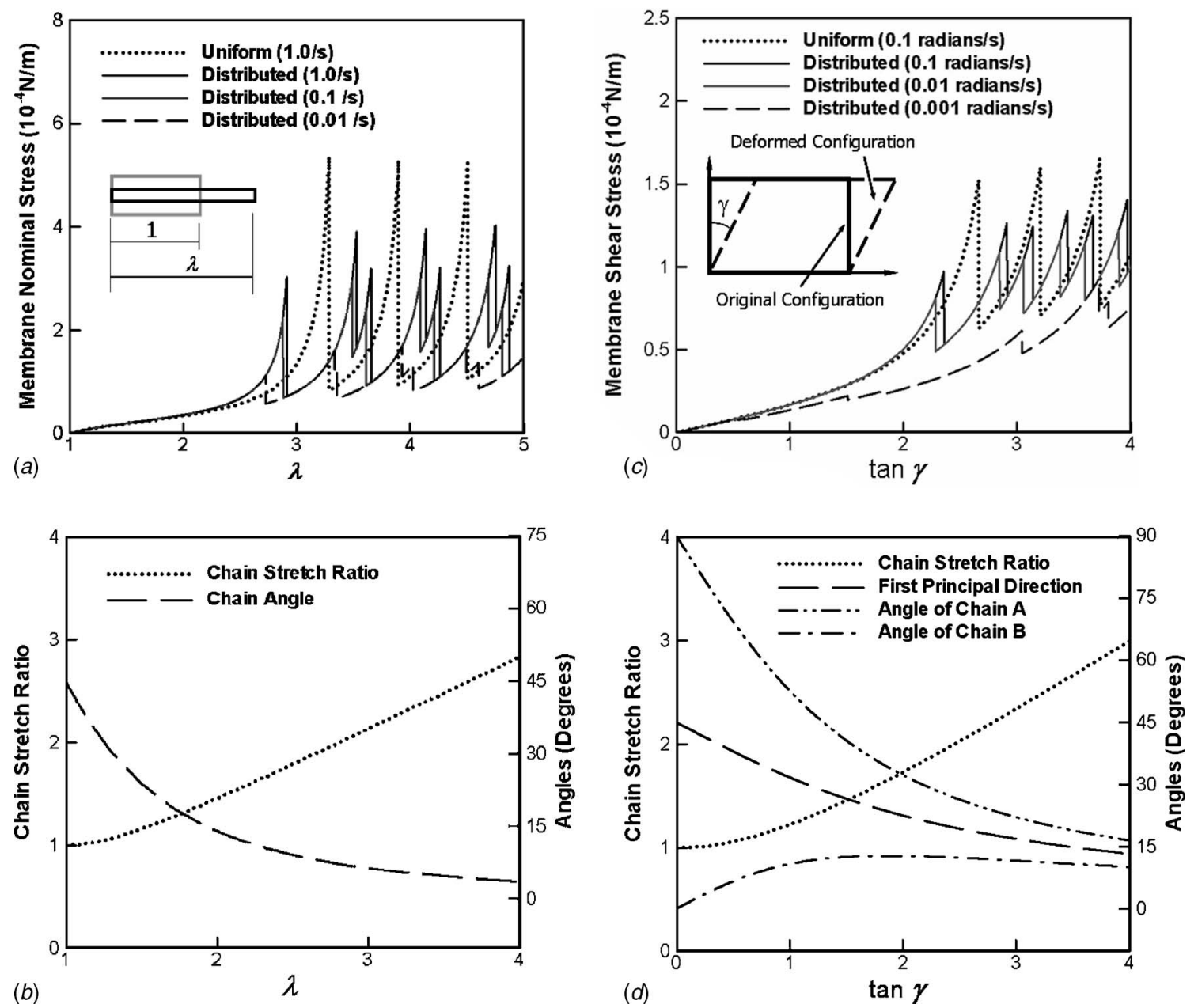

Fig. 6 (a) Uniaxial tensile nominal stress versus stretch behavior of a uniform and distributed network membrane, where the distributed network behavior is also shown at different strain rates; (b) Evolution in chain stretch ratio and chain angle with macroscopic stretch $\lambda$; (c) Membrane shear stress-tan $\gamma$ behavior; (d) Evolution of chain stretch ratio and chain angles with $\tan \gamma$

Figure $6(b)$ shows the evolution in the chain stretch ratio and the molecular orientation during tension. Here, the average molecular chain angle is defined as the angle between the axial stretch direction and the chain direction of (vector connecting the two ends of a chain in the network). The chain angle decreases with increasing sample stretch, capturing the evolution in molecular network orientation with stretch (i.e., the chains align towards the principal stretch direction).

Simple shear results are presented in Fig. 6(c) in terms of the membrane shear stress versus $\tan \gamma$. Figure $6(d)$ shows the corresponding evolutions in the chain stretch ratio and the molecular chain orientations (chain angles) during simple shear. In Fig. 6(d), the first principal direction is expressed in terms of the angle between the first principal direction and the horizontal (1direction); the angles of chains $B$ and $A$ are the angles between the chain and the horizontal direction (1-direction). The chains are found to stretch and orient towards the first principal stretch direction with increasing shear angle where the first principal direction, in turn, aligns towards the horizontal direction as the shear angle $\gamma$ increases.

3.4 Macromolecular Solid Network Model. The threedimensional behavior of a solid composed of a modular macromolecular network is illustrated for two loading conditions: uniaxial tension and equi-biaxial tension. The same single molecule material properties used for the spectrin planar network were adopted to simulate the stress-stretch behavior of the solid.
The chain density is calculated from the 3D network arrangement with $r_{0}=75 \mathrm{~nm}$. Figure $7(a)$ shows the uniaxial tension stressstretch behavior when the network chains are assigned identical initial contour length (dotted line) and when a distribution in initial contour length is considered. The uniform network stressstretch behavior is found to exhibit a similar saw-tooth pattern as the single molecule and membrane behavior. For the distributed network, the stress and stretch at first unfolding are significantly lower and the stress-stretch curve exhibits a smoother character. In equi-biaxial tension (Fig. $7(c)$ ), due to extension in two directions, the chain stretch is $\lambda_{c}=\sqrt{\left(2 \lambda^{2}+\lambda^{-4}\right) / 3}$, and is larger than the chain stretch in uniaxial tension at any given specimen stretch. Therefore, unfolding occurs at a smaller stretch ratio.

The evolutions in the chain stretch ratio and molecular orientation (chain angle) during deformation are shown for uniaxial tension (Fig. 7(b)) and equi-biaxial tension (Fig. $7(d)$ ). The model captures the behavior of the chains stretching and rotating towards the macroscopic principal stretch direction(s) forming an oriented molecular network structure with imposed stretch.

3.5 Network Pre-tension. Note that a non-zero initial end-toend distance $r_{0}$ provides a pretension in the network. Pretension (also referred to as prestress) in various cell structural components is thought to play a significant role in the mechanics of the cell [13]. The constitutive model formulation developed here naturally includes pretension. The effect of pretension is modeled by speci- 

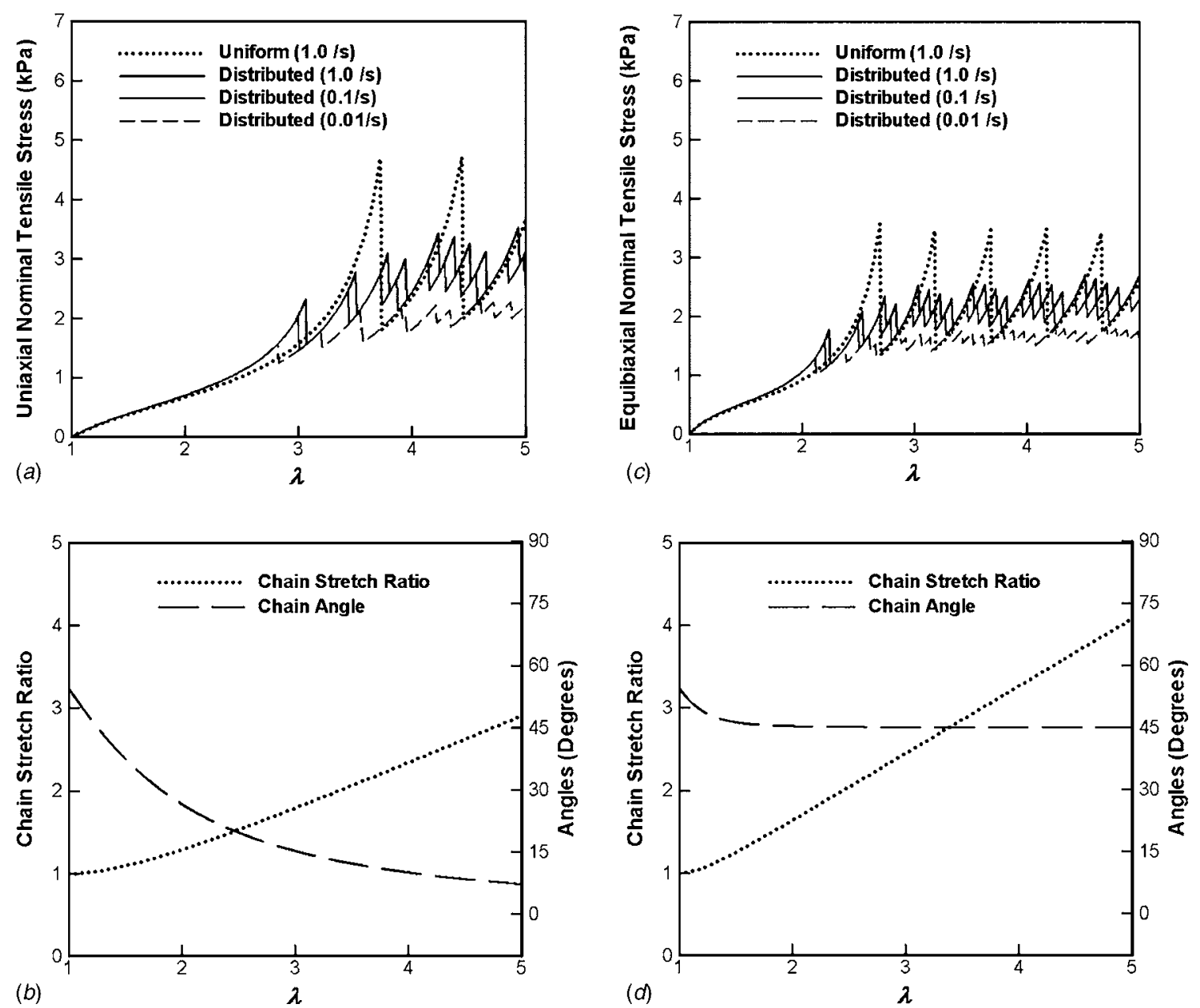

Fig. 7 Eight-chain network model results for: (a) uniaxial tension nominal stress versus stretch behavior; (b) evolution in chain stretch ratio and chain angle with macroscopic stretch during uniaxial tension; (c) equibiaxial tension nominal stress versus stretch behavior; (d) evolution in chain stretch ratio and chain angle with macroscopic stretch during equibiaxial tension

fying the initial end-to-end distances $\left(r_{0}\right)$ of the chains in the network, where $r_{0}$ gives the initial extended (tensioned) state of each chain. A larger $r_{0}$ coincides with larger pretension. Any changes in $r_{0}$ also correspond to changes in the initial chain density for a given network and an increase in $r_{0}$ necessarily corresponds to a decrease in areal chain density $\nu$. Figure 8 depicts the model prediction of the effects of pretension on the uniaxial nominal stress-stretch behavior of a three-dimensional network. Pretension is seen to alter the stress-strain behavior in two significant ways. First, pretension acts to increase the initial modulus of the membrane for the realistic range in pretensions examined here. Although pretension acts to reduce the chain areal density which, by itself, would decrease the initial modulus through the modulus proportionality to $\nu k T$, this reduction is offset by the chain preextension which takes the chains into the high tangent stiffness regime of their force-extension curve and thus provides the predicted overall increase in modulus with increase in pretension. Second, an increase in pretension is found to dramatically decrease the macroscopic stress level and the stretch level required to initiate unfolding and produce the stress plateau region. Furthermore, the stress plateau region becomes smoother with increasing prestress. The effects of pretension on the initial stiffness, the stress and stretch of unfolding, and the plateau will have significant impact on the deformation characteristics during cell functioning, governing the cells ability to deform. In particular, unfolding eliminates the dramatic stiffening behavior and enables

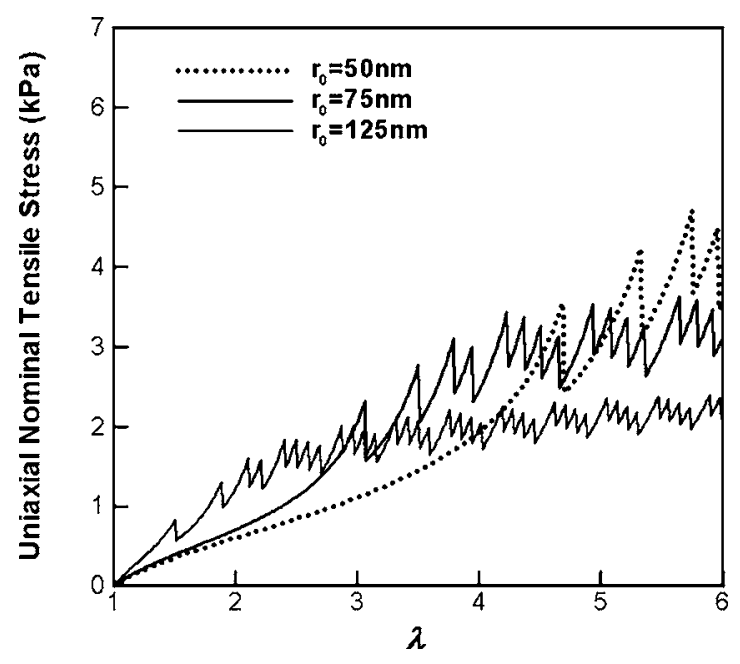

Fig. 8 Effect of pretension on the uniaxial stress-stretch behavior of a three-dimensional network (at the strain rate of $0.1 / \mathrm{s}$ for the distributed network) 
the cell to accommodate large deformations under relatively low and controlled stress levels. One example is the plateau behavior providing a plastic hinge-like behavior to the overall cell bending and folding deformations required for the travel of the red blood cell through the circulatory system.

\section{Conclusion}

Constitutive models for the force-extension behavior of multimolecule strands of modular macromolecules and the finite deformation multi-axial stress-stretch behavior of membranes and solids containing modular macromolecules have been presented. The constitutive model results for strands of multiple molecules demonstrate that a relatively modest distribution in chain structure smoothes out the saw-tooth character of the single molecule forceextension behavior giving a plateau region in the force-extension curve. The plateau corresponds to simultaneous and competing chain stretching and module unfolding events taking place in the different constituent molecular chains. The membrane formulation is considered a two-dimensional network of modular macromolecules. The model specifically accounts for the single molecule force-extension behavior, the initial end-to-end distance of chain segments (the pretension), and the cross-link density; these structural features are all directly measurable quantities. Here, a fourchain network representation of a random surface network was presented; more specific network geometries can also be simulated where, for example, Arslan and Boyce [36] have presented a continuum mechanics constitutive model of triangulated surface networks. The proposed constitutive model was then used to simulate uniaxial and simple shear loading of a representative membrane, demonstrating how single molecule behavior translates into membrane behavior and thus revealing the membrane loading conditions which trigger stretch-induced unfolding. While the model provides the multiaxial stress-stretch behavior of the membrane, it also retains intimate details of the underlying network structure and its evolution with loading including the stretch and force on the constituent molecules, the forces acting on the cross-link sites, the orientation of the molecules and the links within the molecules, as well as the number of folded and unfolded modules along the chain. The same approach was utilized in formally constructing a constitutive model for threedimensional networks, again building from the single molecule force-extension behavior together with network structure and allowing the same level of structural detail and evolution to be captured and monitored. This framework enables parametric study of the influence of microstructural features on the stress-stretch behavior. As an illustration, the effects of pretension on the stressstretch behavior were shown, revealing the strong effects on the initial modulus as well as on the stress and stretch levels required to induce unfolding. The rate effects on the stress-stretch behavior of the networks due to the rate dependence of unfolding are also captured by the model. Additional rate effects such as those due to viscoelastic phenomena arising from intermolecular interactions as well as those due to fluid flow are not included in the model, but can be included following established nonlinear viscoelastic modeling approaches (e.g., [37-40]). The proposed constitutive models for the membrane and the solid are a starting point for understanding the role of modular protein mechanical behavior in issues of cell mechanics as well as in issues of protein-rich biological materials which may act as load transfer and/or dissipative agents in biological composite structures and fibers.

\section{Acknowledgment}

This research was supported by the U. S. Army through the Institute for Soldier Nanotechnologies, under Contract No. DAAD-19-02-D0002 with the U.S. Army research office. The content does not necessarily reflect the position of the government and no official endorsement should be inferred.

\section{Appendix A: Worm-Like Chain Equations}

The mechanical behavior of biomolecules has also been successfully modeled using the WLC model. The WLC model originates from the Kratky-Porod chain model $[16,17]$. The WLC model, as approximated by Marko and Siggia [18], gives the stored energy during axial stretch to be:

$$
u=\frac{k_{B} T}{A} L\left[\frac{1}{2}\left(\frac{r}{L}\right)^{2}+\frac{1}{4(1-r / L)}-\frac{1}{4}\left(\frac{r}{L}\right)\right]
$$

where $r$ is the end-to-end distance, $A$ is the persistence length. Below we provide the constitutive model equations using WLC as the molecule behavior for the single molecule, multi-molecule strand, membrane network, and three-dimensional network models.

Single Molecule Force-Extension:

$$
f_{c}=\frac{k_{B} T}{A}\left(\frac{r}{L}+\frac{1}{4(1-r / L)^{2}}-\frac{1}{4}\right)
$$

Multi-Molecule Strand Force-Extension:

$$
F=\frac{k_{B} T}{A} \sum_{i=1}^{k}\left(\frac{r}{L_{i}}+\frac{1}{4\left(1-r / L_{i}\right)^{2}}-\frac{1}{4}\right)
$$

Membrane Four-Chain Network Model Strain energy density function:

$$
\tilde{U}=\nu k_{b} T N\left[\frac{1}{2}\left(\frac{\lambda_{c} r_{0}}{L}\right)^{2}+\frac{1}{4\left(1-\lambda_{c} r_{0} / L\right)}-\frac{\lambda_{c} r_{0}}{4 L}-c\right]
$$

Membrane Cauchy stress:

$$
\tilde{\boldsymbol{\sigma}}=\nu k_{B} T \frac{r_{0} N}{2 \lambda_{c} L}\left[\frac{\lambda_{c} r_{0}}{L}+\frac{1}{4\left(1-\lambda_{c} r_{0} / L\right)^{2}}-\frac{1}{4}\right] \mathbf{B}_{2 \mathrm{D}}+\tilde{h} \mathbf{I}_{2 \mathrm{D}}
$$

Three-dimensional Eight-Chain Network Model strain energy density:

$$
\bar{U}=\nu k_{B} T N\left[\frac{1}{2}\left(\frac{\lambda_{c} r_{0}}{L}\right)^{2}+\frac{1}{4\left(1-\lambda_{c} r_{0} / L\right)}-\frac{\lambda_{c} r_{0}}{4 L}-c\right]
$$

Eight-Chain Network Model Cauchy stress:

$$
\boldsymbol{\sigma}=\nu k_{B} T \frac{r_{0}}{3 \lambda_{c}} \frac{N}{L}\left[\frac{\lambda_{c} r_{0}}{L}+\frac{1}{4\left(1-\lambda_{c} r_{0} / L\right)^{2}}-\frac{1}{4}\right] \mathbf{B}-p^{*} \mathbf{I}
$$

Figure 9 shows the comparison between the FJC model and WLC model for the case of simple tension of a membrane; FJC and WLC models give similar results for the membrane behavior.

\section{Appendix B: Model Procedure for Force Extension With Unfolding}

The probability of unfolding is evaluated at each increment in extension and accumulated with time until reaching a critical value; the steps are summarized here:

1. Initialize the probability of unfolding: $d p_{(0)}=0.0$,

2. Apply an increment in extension; compute the force using either the FJC or WLC model,

3. Compute the unfolding frequency, $\omega_{u}$, using Eq. (3). Determine the probability of unfolding for the time up to this increment: $d p_{(t)}=m_{t} \omega_{u} \Delta t+d p_{(t-1)}$, where $\Delta t$ is time increment and $m_{t}$ is the number of folded domains.

4. (a) If $d p_{(t)}>p_{c r}$, unfolding occurs. The contour length of the protein is increased by $q l$, where $q$ is the number of Kuhn segments in a folded module and $l$ is the length of a Kuhn segment. Set $d p_{(t)}=0.0$. Go to step 2 .

(b) If $d p_{(t)}<p_{c r}$, unfolding does not occur. Go to step 2. 


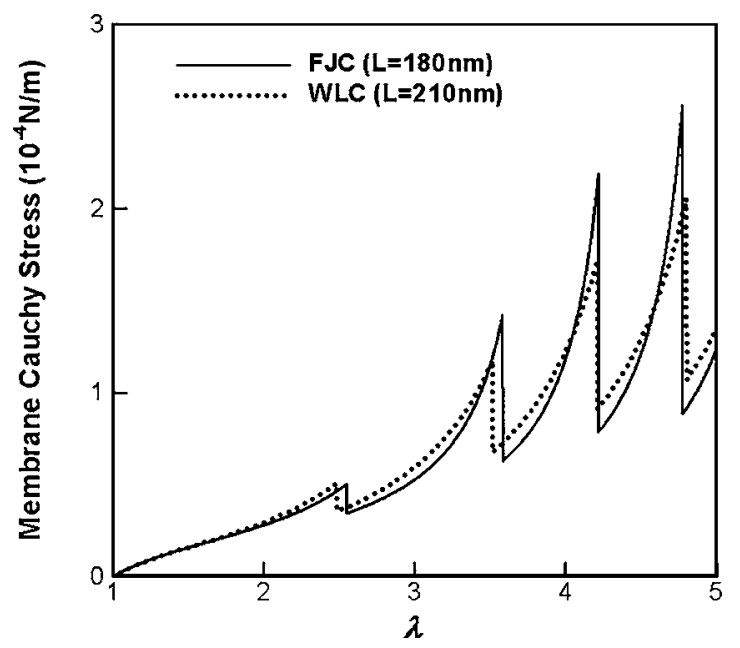

Fig. 9 Comparison of FJC model and WLC model predictions for uniaxial membrane nominal stress-strain behavior

\section{Appendix C: Formulation of Stress-Strain Relationship From Strain Energy Density Function}

Formulation of the stress-strain behavior follows that of Qi and Boyce [41]. From the second law of thermodynamics, the Clausius Duhem inequality for an isothermal process can be written as:

$$
\frac{1}{2} \mathbf{S} \cdot \dot{\mathbf{C}}-\dot{\bar{U}} \geqslant 0
$$

as expressed in terms of the work conjugate second Piola Kirchhoff stress $\mathbf{S}=\operatorname{det}(\mathbf{F}) \mathbf{F}^{-1} \boldsymbol{\sigma} \mathbf{F}^{T-1}$ and the right Cauchy Green tensor $\mathbf{C}=\mathbf{F}^{T} \mathbf{F} \cdot \bar{U}$ is the strain energy density function, and in the proposed model, is defined to be a function of $I_{1}$ and the state variable of the number of persistent segments along the chain, $N$

$$
\bar{U}=\bar{U}\left(I_{1}, N\right)
$$

From Eq. (C2),

$$
\dot{\bar{U}}=\frac{\partial \bar{U}}{\partial I_{1}} \frac{\partial I_{1}}{\partial \mathbf{C}} \cdot \dot{\mathbf{C}}+\frac{\partial \bar{U}}{\partial N} \dot{N}
$$

Inequality $(\mathrm{C} 1)$ gives

$$
\left(\frac{1}{2} \mathbf{S}-\frac{\partial \bar{U}}{\partial I_{1}} \frac{\partial I_{1}}{\partial \mathbf{C}}\right) \cdot \dot{\mathbf{C}}-\frac{\partial \bar{U}}{\partial N} \dot{N} \geqslant 0
$$

For an arbitrary deformation,

$$
\mathbf{S}=2 \frac{\partial \bar{U}}{\partial I_{1}} \frac{\partial I_{1}}{\partial \mathbf{C}}
$$

and

$$
-\frac{\partial \bar{U}}{\partial N} \dot{N} \geqslant 0
$$

For an incompressible material, Eq. (C5a) gives the second Piola Kirchhoff stress, $\mathbf{S}=2 \frac{\partial \bar{U}}{\partial I_{1}} \frac{\partial I_{1}}{\partial \mathbf{C}}-p \mathbf{C}^{-1}$. Thus, the Cauchy stress is $\boldsymbol{\sigma}$ $=2 \partial \bar{U} / \partial I_{1} \mathbf{B}-p^{*} \mathbf{I}$. Inequality $(\mathrm{C} 5 b)$ shows unfolding a module is a dissipative process; analogous arguments would hold for refolding but are not addressed in this paper.

\section{References}

[1] Yan, Y., Winograd, E., Viel, A., Cronin, T., Harrison, S. C., and Branton, D., 1993, "Crystal Structure of the Repetitive Segments of Spectrin," Science, 262(5142), pp. 2027-2030.

[2] Rief, M., Gautel, M., Oesterhelt, F., Fernandez, J. M., and Gaub, H. E., 1997,
"Reversible Unfolding of Individual Titin Immunoglobulin Domains by AFM," Science, 276, pp. 1109-1112.

[3] Oberhauser, A. F., Marszalek, P. E., Erickson, H. P., and Fernandez, J. M., 1998, "The Molecular Elasticity of the Extracellular Matrix Protein Tenascin," Nature (London), 393, pp. 181-185.

[4] Fisher, T. E., Oberhauser, A. F., Carrion-Vazquez, M., Marszalek, P. E., and Fernandez, J. M., 1999, "The Study of Protein Mechanics With the Atomic Force Microscope," TIBS, 24, pp. 379-384.

[5] Rief, M., Pascual, J., Saraste, M., and Gaub, H. E., 1999, "Single Molecule Force Spectroscopy of Spectrin Repeats: Low Unfolding Forces in Helix Bundles," J. Mol. Biol., 286, pp. 553-561.

[6] Smith, B. L., Schäffer, T. E., Viani, M., Thompson, J. B., Frederick, N. A Kindt, J., Belcher, A., Stucky, G. D., Morse, D. E., and Hansma, P. K., 1999, "Molecular Mechanistic Origin of the Toughness of Natural Adhesives, Fibres and Composites," Nature (London), 399, pp. 761-763.

[7] Li, H. B., Carrion-Vazquez, M., Oberhauser, A. F., Marszalek, P. E., and Fernandez, J. M., 2000, "Point Mutations Alter the Mechanical Stability of Immunoglobulin Modules," Nat. Struct. Biol., 7, pp. 1117-1120.

[8] Minajeva, A., Kulke, M., Fernandez, J. M., and Linke, W. A., 2001, "Unfolding of Titin Domains Explains the Viscoelastic Behavior of Skeletal Myofibrils," Biophys. J., 80, pp. 1442-1451.

[9] Mohandas, N., and Evans, E., 1994, "Mechanical Properties of the Red Cell Membrane in Relation to Molecular Structure and Genetic Defects," Annu. Rev. Biophys. Biomol. Struct., 23, pp. 787-818.

[10] Jackson, A. P., Vincent, J. F. V., and Turner, R. M., 1988, "The Mechanical Design of Nacre," Proc. R. Soc. London, Ser. B, 234, pp. 415-440.

[11] Qi, H. J., Bruet, B. J. F., Palmer, J. S., Ortiz, C., and Boyce, M. C., 2005, "Micromechanics and Macromechanics of the Tensile Deformation of Nacre", Chapter in Mechanics of Biological Tissues, Proceedings IUTAM, edited by G. A. Holzapfel, and R. W. Ogden, Springer-Verlag, Graz, Austria.

[12] Gosline, J. M., Guerette, P. A., Ortlepp, C. S., and Savage, K. N., 1999, "The Mechanical Design of Spider Silks: From Fibroin Sequence to Mechanical Function," J. Exp. Biol., 202, pp. 3295-3303.

[13] Zhu, C., Bao, G., and Wang, N., 2000, "Cell Mechanics: Mechanical Response, Cell Adhesion, and Molecular Deformation," Annu. Rev. Biomed. Eng., 2, pp. 189-226.

[14] Kuhn, W., and Grun, F., 1942, "Beziehungen Zwischen Elastischen Konstanten Und Dehnungsdoppelbrechung Hochelastischer Stoffe,” Kolloid-Z., 101, pp. 248-271.

[15] Treloar, L. R. G., 1958, The Physics of Rubber Elasticity, Clarendon, Oxford.

[16] Kratky, O., and Porod, G., 1949, "Röntgenuntersuchung Gelöster Fadenmoleküle," Recl. Trav. Chim. Pays-Bas, 68, pp. 1106-1122.

[17] Fixman, M., and Kovac, J., 1973, "Polymer Conformational Statistics. III Modified Gaussian Models of Stiff Chains," J. Chem. Phys., 58, pp. 1564 1568

[18] Marko, J. F., and Siggia, E. D., 1995, “Stretching DNA,” Macromolecules, 28, pp. 8759-8770.

[19] Eyring, H., 1936, "Viscosity, Plasticity and Diffusion as Examples of Absolute Reaction Rates,” J. Chem. Phys., 4, pp. 283-291.

[20] Bell, G. I., 1978, "Models for the Specific Adhesion of Cells to Cells," Science, 200, pp. 618-627.

[21] Lu, H., Isralewitz, B., Krammer, A., Vogel, V., and Schulten, K., 1998, "Unfolding of Titin Immunoglobulin Domains by Steered Molecular Dynamics Simulation," Biophys. J., 75, pp. 662-671.

[22] Gao, M., Lu, H., and Schulten, K., 2002, "Unfolding of Titin Domains Studied by Molecular Dynamics Simulations," J. Muscle Res. Cell Motil., 23, pp. 513-521.

[23] Byers, T. J., and Branton, D., 1985, "Visualization of the Protein Associations in the Erythrocyte Membrane Skeleton," Proc. Natl. Acad. Sci. U.S.A., 82, pp. 6153-6157.

[24] Liu, S., Derick, L. H., and Palek, J., 1987, "Visualization of the Hexagonal Lattice in the Erythrocyte Membrane Skeleton,” J. Cell Biol., 103, pp. 527536.

[25] Arruda, E. M., and Boyce, M. C., 1993, “A Three-Dimensional Constitutive Model for the Large Stretch Behavior of Rubber-Elastic Materials," J. Mech. Phys. Solids, 41, pp. 389-412.

[26] Skalak, R., Tozeren, A., Zarda, R. P., and Chien, S., 1973, "Strain Energy Function of Red Blood Cell Membranes," Biophys. J., 13, pp. 245-264.

[27] Evans, E. A., 1973, "A New Material Concept for the Red Blood Cell Membrane," Biophys. J., 13, pp. 926-940.

[28] Evans, E. A., and Hochmuth, R. M., 1977, "A Solid-Liquid Composite Model of Red Cell Membrane," J. Membr. Biol., 30, pp. 351-362.

[29] Dao, M., Lim, C. T., and Suresh, S., 2003, "Mechanics of the Human Red Blood Cell Deformed by Optical Tweezers," J. Mech. Phys. Solids, 51, pp. 2259-2280.

[30] Mills, J. P., Qie, L., Dao, M., Lim, C. T., and Suresh, S., 2004, "Nonlinear Elastic and Viscoelastic Deformation of the Human Red Blood Cell With Optical Tweezers," Mech. Chem. Biosyst., 1, pp. 169-180.

[31] Boyce, M. C., and Arruda, E. M., 2000, "Constitutive Models of Rubber Elasticity: A review," Rubber Chem. Technol., 73, pp. 504-523.

[32] Bischoff, J., Arruda, E. M., and Grosh, K., 2002, "Orthotropic Hyperelasticity in Terms of an Arbitrary Molecular Chain Model," J. Appl. Mech., 69, pp 198-201.

[33] Bischoff, J., Arruda, E. M., and Grosh, K., 2002, "A Microstructurally Based Orthotropic Hyperelastic Constitutive Law,” J. Appl. Mech., 69, pp. 570-579.

[34] Bischoff, J., Arruda, E. M., and Grosh, K., 2001, "A New Constitutive Mode for the Compressibility of Elastomers at Finite Deformation," Rubber Chem. 
Technol., 74, pp. 541-559.

[35] Boey, S. K., Boal, D. H., and Discher, D. E., 1998, "Simulations of the Erythrocyte Cytoskeleton at Large Deformation. I. Microscopic Models," Biophys. J., 75, pp. 1573-1583.

[36] Arslan, M., and Boyce, M. C., 2005, "Constitutive Modeling of the Finite Deformation Behavior of Membranes Possessing a Triangulated Network Microstructure,” ASME J. Appl. Mech., 73, pp. 536-543.

[37] Bergstrom, J. S., and Boyce, M. C., 1998, "Constitutive Modelling of the Large Strain Time-Dependent Behavior of Elastomers," J. Mech. Phys. Solids, 46, pp. 931-954.
[38] Bergstrom, J. S., and Boyce, M. C., 2000, "Large Strain Time-Dependent Behavior of Filled Elastomers," Mech. Mater., 32, pp. 627-644.

[39] Reese, S., and Govindjee, S., 1998, "A Theory of Finite Viscoelasticity and Numerical Aspects," Int. J. Solids Struct., 35, pp. 3455-3482.

[40] Qi, H. J., and Boyce, M. C., 2005, "Stress-Strain Behavior of Thermoplastic Polyurethane," Mech. Mater., 37, pp. 817-830.

[41] Qi, H. J., and Boyce, M. C., 2004, "Constitutive Model for Stretch-Induced Softening of the Stress-Stretch Behavior of Elastomeric Materials," J. Mech. Phys. Solids, 52, pp. 2187-2205. 